\title{
Application of pore network modeling in deep bed filtration analysis
}

\author{
Ali Shabani ${ }^{1}\left(\mathbb{0} \cdot\right.$ Davood Zivar $^{2} \cdot$ Hamid Reza Jahangiri $^{3} \cdot$ Abbas Shahrabadi $^{4}$
}

Received: 4 November 2019 / Accepted: 17 August 2020 / Published online: 19 August 2020

(c) Springer Nature Switzerland AG 2020

\begin{abstract}
Particle invasion in porous media is an important phenomenon that could lead to formation damage during different operations, such as waterflooding, workover, and drilling. In this paper, a 3D pore network model coupled with a particle tracking method was developed to investigate particle retention and permeability reduction of a pore network system. The proposed model considers the effect of hydraulic drag, gravity, and friction forces. Three mechanisms, including surface deposition, straining, and bridging, have been considered in the development of the proposed pore network model. The results of the proposed model show good agreement with experimental data. A sharp permeability reduction is observed in the early time of the injection, which indicates the blockage of the small radius throats by particles, as well as unstable fluid flow due to the distribution of the particles. Moreover, the number of throats with a small radius and different contributing mechanisms cause the discontinuous decrease of the porous media permeability. The proposed pore network modeling demonstrates that a small section of the pore network can reproduce the results of the experiment, and a big pore network that is too time and cost consuming is not required.
\end{abstract}

Keywords Deep bed filtration · Pore network modeling · Bridging · Straining · Surface deposition

\section{List of symbols}

$F_{H} \quad$ Hydrodynamic force $(\mathrm{N})$

$F_{B} \quad$ Buoyancy force $(\mathrm{N})$

$F_{W} \quad$ Weight of particle (N)

$F_{f} \quad$ Friction force $(\mathrm{N})$

$F_{F} \quad$ Resultant of forces in the opposite direction of $\quad \rho_{f}$ particle movement

$K \quad$ Permeability of the medium $\left(\mathrm{m}^{2}\right)$

$K_{0} \quad$ Initial permeability of the medium $\left(\mathrm{m}^{2}\right)$

$L_{i j} \quad$ Length of the throat connecting pore $\mathrm{i}$ to pore $\mathrm{j}$ (m)

$P \quad$ Pressure $(\mathrm{Pa})$

$r_{\text {blocking }}$ Blocking radius of the throat $(\mathrm{m})$

$r_{e} \quad$ Effective radius of the throat $(\mathrm{m})$

$r_{i} \quad$ Initial radius of the throat $(\mathrm{m})$

$r_{p} \quad$ Particle radius $(\mathrm{m})$

$\beta \quad$ An arbitrary integer number between 3 and 7
$U_{f} \quad$ Magnitude of the fluid velocity in the throat $(\mathrm{m} / \mathrm{s})$

$U_{p} \quad$ Magnitude of the particle velocity in the throat $(\mathrm{m} / \mathrm{s})$

$\mu \quad$ Viscosity of the injected liquid (cp)

$\rho_{f} \quad$ Density of injected fluid ( $\mathrm{gr} / \mathrm{cc}$ )

$\rho_{p} \quad$ Density of particles ( $\mathrm{gr} / \mathrm{cc}$ )

$\varphi \quad$ Porosity of the porous media

$\varphi_{t} \quad$ Porosity of the throat

$\theta \quad$ The angle between the throat and the horizon $y \quad$ Coefficient for blocking radius calculation

Ali Shabani, alishabani.sharif@gmail.com | 'Department of Chemical and Petroleum Engineering, Sharif University of Technology, Tehran, Iran. ${ }^{2}$ Centre of Research in Enhanced Oil Recovery, Universiti Teknologi PETRONAS, Perak, Malaysia. ${ }^{3}$ Department of Chemical and Petroleum Engineering, Iran University of Science and Technology, Tehran, Iran. ${ }^{4}$ Upstream Technologies Research Center, Research Institute of Petroleum Industry (RIPI), West Blvd. of Azadi Sports Complex, Tehran, Iran. 


\section{Introduction}

During different operations, such as water purification, contaminants dispersion, and drilling mud invasion, suspended solid particles are transported and filtered in porous media [1]. In this light, a suspended particle may be a separated piece of reservoir rock, a foreign particle already exist in the injection fluid or a scale that is generated in the reservoir due to the interaction of the injected fluid with formation water. The invasion and filtration of particles are called the deep bed filtration (DBF). Studies have found that DBF could also happen in water flooding and/or wellbore workover operation, when the injected water is not filtered and contains a massive amount of particles or ions that precipitate and deposit in porous media.

The researchers have studied DBF experimentally [2-6] and most studies have reported the permeability reduction of the core samples versus time. For example, Baghdikian et al. [6] injected clay suspensions in the Ottawa sand pack to study the effect of ionic strength, concentration, and flow rate of the injected fluid. Moghadasi et al. [4] injected inert aluminum particles in a cylindrical porous media made from glass bead. Mentioned studies reported that parameters, such as flow rate, particle concentration, and the initial permeability of porous media, could affect the permeability of the medium. They also found that apart from the mentioned parameters, the particles that are initially present in the porous medium could affect the results of experiments [4]. Another study by Alem et al. [7] examined the effect of hydrodynamic force (fluid velocity) on particle deposition in different axial distances from the fluid inlet. It was shown that lower injection velocities enhance the deposition of suspended particles, due to the reduction in hydrodynamic forces [7].
According to the results of recent studies $[1,8]$, traveling particles through the pores and throats of the porous media could get stuck with one of the underlying mechanisms, however, smaller size particles may cross the pores without getting stuck.

Pore plugging) some of the particles may bridge or plug the pores when traveling through pores and throats as presented in Fig. 1a. These particles severely reduce the permeability and porosity of the porous medium.

Surface deposition) other injected particles might be deposited onto the surface of pores by mechanisms presented in Fig. 1b. These particles reduce the porosity while causing an insignificant effect on the permeability of the porous media.

The continuity equation of particles is widely used for simulation of particle transport and filtration in the porous media, where it was used by many researchers to simulate experimental results of DBF [7-12]. Each study has tried to develop the continuity equation of particles by defining: (1) matching parameters or (2) capturing rate equations for permeability and particles $[9,13]$. While the results of continuity equations showed a good agreement with experimental results, the story behind of pore-scale interactions did not describe well.

Many researchers have tried to simulate DBF using pore network modeling [5]. A pore network is a lattice of pores, which are connected to each other via the throats [14]. The lattice could be in either 2D or 3D geometry. Although calculation problems might occur, the pore network modeling is still widely used for pore-scale studies. Todd et al. [5] performed a DBF study using a pore network by injecting the micron size particles of aluminum into the sandstone cores in order to study the behavior of pore blockage by aluminum particles [5]. Figure 2 presents a throat, which is bridged by aluminum particles.

On the other hand, Todd et al. [5] had largely ignored the contribution of the bridging mechanism and the
Fig. 1 The schematic of the main mechanisms of particle capturing in porous media [8]

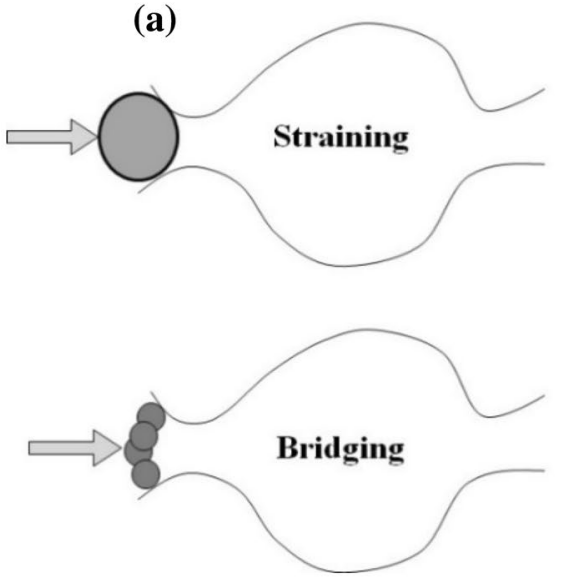

(b)

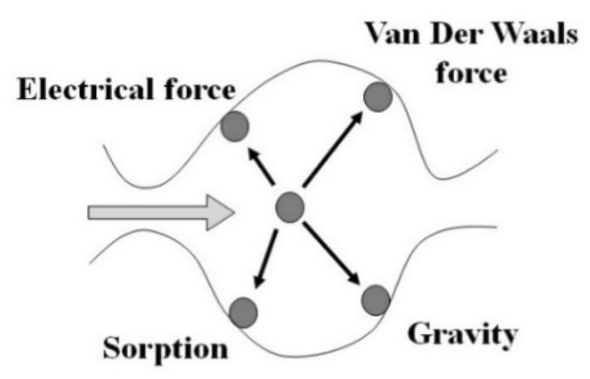




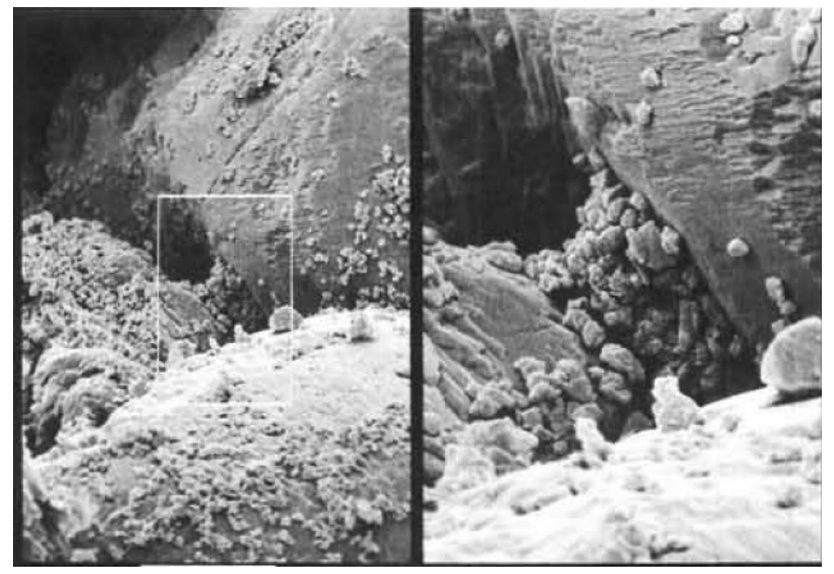

Fig. 2 Pore bridging and surface deposition reported by Todd et al. [5]

random location of pores in the presented model. A simple random number was used for the selection of new throat for particles when the particle is in the pore [5]. Subsequently, Rege and Fogler [15] presented a method with higher accuracy for the selection of new throat based on the fluid velocity of each throat. Other researchers have considered the particle movement in the throats [1, 16, 17]. Yang and Balhoff [1] considered the effect of fluid velocity profile in the cross-section of throats. They used a regular lattice for pore network and the bridging mechanism was not contributed in the introduced model [1].

In comparison with straining, the bridging mechanism is the main mechanism that causes a severe permeability reduction in porous media. However, this mechanism has been excluded by most of the researchers in pore network studies. This study tried to present a pore network model for simulation of DBF in a core scale that considers three mechanisms of surface deposition, straining, and bridging. In the proposed model, particle path selection follows the model proposed by Rege and Fogler [15]. A constant flow boundary was assigned to the model. Moreover, a random 3D pore location was selected for the model. In addition, this study proposes a method for applying the forces on particles that considers the effect of hydraulic drag, gravity, and friction forces. In the proposed network model instead of focusing on the complicated transverse movement of a particle in throats, it was tried to present a real particle injection situation. The results of the proposed network model show a good agreement with the permeability decline of experiments. The structure of the paper is as follows: Section two presents the pore network model. Section three describes the particle movement in the pore network model. Results and discussions of the pore network model are presented in section four, and section five presents the conclusions.

\section{Pore network model}

As stated before, a pore network is a lattice of pores, which are connected to each other using throats [14]. In the real case, the morphology of pores and throats are complicated, and it is time-consuming to simulate such a complex system of pores and throats by a numerical solution. In the presented pore network model, the system is simplified into interconnected nodes (pores), which are connected to each other using bonds (throats). A random distribution of the interconnected nodes (pores) is used in order to distribute the pores in 3D geometry. The number of pores connected to a single pore (by throats) is known as the coordination number. The present study uses the coordination number of 4 , which reflects that every single pore is connected with 4 adjacent pores [18]. Furthermore, as shown in Fig. 5, the mean diameter of grains of porous media is selected as the average distance between the pores (throats length). A mass conservation equation can be derived for each pore as presented below:

$\sum_{j=1}^{n} \frac{987 \pi r_{i j}^{4}}{8 \mu}\left(\frac{P_{i}-P_{j}}{L_{i j}}\right)=0$

where $r_{i j}$ and $L_{i j}$ are radius and length of the throat connecting pore $i$ and $\mathrm{j}, \mu$ is the fluid viscosity, $P_{i}$ and $P_{j}$ are the pressures in pore $\mathrm{i}$ and pore j. Solving Eq. 1 for each pore represents the pressure at pores and the flux (velocity) in throats. The initial value of the throats radius is set according to the normal distribution function. Then the mean radius of throats is changed to match the permeability of studied porous media. In this light, the pore network with a specified mean radius of throats, throats length, and the spatial distribution of pores is the representative of the porous media.

The effect of surface deposition mechanism on the permeability alteration of the pore network is not significant, while the straining and bridging reduce permeability dramatically. Due to fact that the mentioned mechanisms are able to reduce the permeability of the pore network and consequently increase the resistivity against the fluid flow when the particles are injecting with a constant rate, the filed pressure needs to be updated in each time step. On the other hand, in each step, all the particles are moving simultaneously, and the interaction between moving particles in the throats is neglected.

To apply constant flow boundary conditions, new inlet pressure is determined for the pore network, then, it is required to recalculate the pressure field. Meanwhile, to inject a constant concentration of the particles into the inlet face of the pore network, a certain number of particles need to be injected into the interface of the pore 
network after several time steps. It is worth mentioning that MATLAB software is used for numerical computing.

\section{Particle movement}

A mechanism for particle deposition in throats is presented based on the consequent of forces acting on the particle. It should be mentioned that this study assumes there is no interaction between particles. A particle selects a new throat based on the mechanism explained by French [19]. When a particle is presented in the pore, and it's required to choose a new path, the straining of bridging mechanisms may occur. These particle capturing mechanisms have been investigated below.

\subsection{Surface deposition mechanism}

The main forces acting on a particle in a throat are hydrodynamic, gravity, and friction forces. The hydrodynamic force always moves the particle forward in the throats. In this regard, gravity could either agree or disagree with the direction of particle movement. The friction force always disagrees with particle movement direction in the throat. The friction force includes mechanical friction (kinetic or rolling friction force), van der Waals, and electrical forces. However, the effect of van der Waals and electrical forces are neglected in this study. Figure 3 illustrates the forces acting on a particle in the pore network.

In Fig. $3, F_{H}$ is the hydrodynamic force, $F_{B}$ is the buoyancy force, $F_{W}$ is the weight of particle and $F_{f}$ is the friction force. When the particle enters a throat, a random number is generated between 0 and 1. If this number is less than 0.5 , it is assumed that the particle is moving on the throat surface, and if it is greater than 0.5 , it is assumed that the particle is moving at the fluid bulk. If the particle is moving on the throat surface, another random number

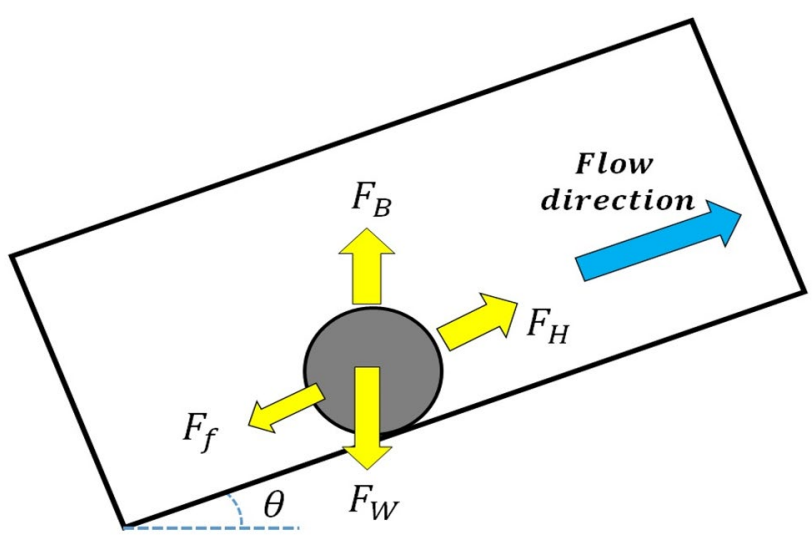

Fig. 3 The schematic of acting forces on a particle is generated. If this number is less than 0.5 , it is assumed that the particle is rolling on the throat surface, and if it is greater than 0.5 , it is assumed that the particle is drawing on the throat surface. Table 1 shows the variation of friction force in these 3 situations.

where, $x$ is the rolling friction factor $(x=0.0001)$ and $z$ is the sliding friction factor $(z=0.1)$ [20]. The hydrodynamic force is calculated according to the equation presented below:

$F_{H}=6 \pi \mu r_{p}\left(U_{f}-U_{p}\right)$

In this equation, $r_{p}$ is the radius of the particle, $U_{f}$ is the fluid velocity and $U_{p}$ is the particle velocity. The resultant of forces in the opposite direction of particle movement is shown by $F_{F}$ as presented below:

$F_{F}=F_{f}+\left(F_{W}-F_{B}\right) \sin (\theta)$

At equilibrium condition, we have $F_{H}=F_{F}$, so by merging Eqs. 2 and 3, the particle velocity is calculated based on Eq. 4.

$U_{p}=U_{f}-\frac{F_{f}+\left(F_{W}-F_{B}\right) \sin (\theta)}{6 \pi \mu r_{p}}$

If $U_{p} \leq 0$, the particle deposits on the throat's surface and surface deposition occur. In this condition, the porosity and the effective radius of throat reduce according to the equation presented below [21]:

$r_{e}=r_{i} \sqrt{\varphi_{t}}$

where $r_{e}$ is the effective radius of the throat, $r_{i}$ is the initial radius of the throat, and $\varphi_{t}$ is the throat porosity. As mentioned before, the permeability reduction of the throats during the surface deposition mechanism is insignificant. The surface deposition mechanism is shown in Fig. 4.

\subsection{Straining mechanism}

The particle will be trapped in the throat where the particle's radius is larger than the radius of the throat. When straining occurs in a throat, it is assumed that the radius of the throat will decrease by 95 percent. The assumption of a 95 percent reduction of throat radius is supported by optimizing the pore network model results with the

Table 1 Friction force acting on a particle

\begin{tabular}{ll}
\hline The friction force & Particle situation \\
\hline$F_{f}=0$ & Particle is moving at the fluid bulk \\
$F_{f}=\left(F_{W}-F_{B}\right) \cos (\theta) x$ & Particle is rolling on the throat surface \\
$F_{f}=\left(F_{W}-F_{B}\right) \cos (\theta) z$ & Particle is drawing on the throat surface \\
\hline
\end{tabular}


Fig. 4 Effective radius of the throat after particles deposition [20]

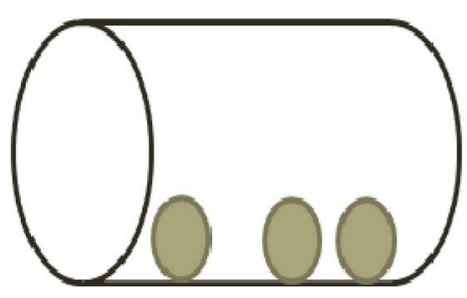

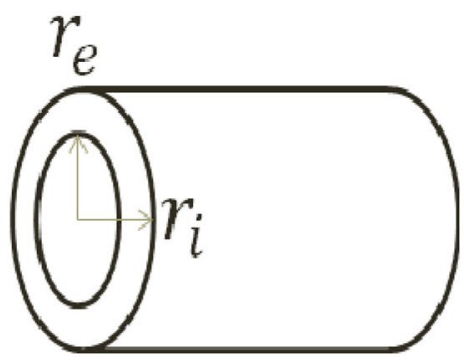

experimental data. In addition, due to the irregular shape of the particles and throats, the possibility of a $100 \%$ blockage of a throat by particles is low. Therefore, the chosen value $(95 \%)$ is within the range of $0 \%$ (the throat radius is fully open to flow) to $100 \%$ (the throat radius is fully blocked).

It is important to note that in real pores and throats systems, as shown in Fig. 5, the radius of a throat is not uniform. As the throat radius is smaller in some sections compared to other sections, the thinner section will have a higher chance of straining and bridging compared to the thicker sections. Hence, it is closer to the reality to define a blocking radius for the smaller throat than the effective radius of the throat. This blocking radius will be compared with particle radius to identify the presence of straining or bridging.

$r_{\text {blocking }}=y r_{e} 0<y<1$

where $r_{\text {blocking }}$ is the blocking radius of the throat, $r_{e}$ is the effective radius of the throat, and $y$ is the coefficient for blocking radius calculations. It should be mentioned that the value of $y$ is considered to be 0.95 .

\subsection{Bridging mechanism}

When a group of particles (in the same time step) want to enter a throat, two conditions have to be met. In this light, the bridging will occur at the throat entrance if the two underlying conditions (Inequality 7 and 8 ) are met.

$n \pi r_{p}^{2}>2 \pi r_{\text {blocking }}^{2}$

$r_{p}>\beta r_{\text {blocking }}$

where $r_{p}$ is the particle radius, $n$ is the number of particles, and $\beta$ is an arbitrary integer number between 3 and 7 [22], which is considered as equal to 3 in this study. Inequality 7 shows that the total area of particle cross-section must be two times bigger than the area of the throat cross-section. Similar to the straining mechanism, it is assumed that the radius of the throat will be reduced when there is a 95 percent blockage as the bridging never blocks the pores completely. Due to the considerable permeability reduction in the bridging mechanism, the pressure field of the pore network is recalculated in the next time step of simulation.

\section{Results and discussion}

An experiment of Moghadasi et al. [4] study is selected to verify the proposed pore network. They injected inert aluminum particles into a glass bead in order to study the effect of fluid velocity [4]. In the experiment, the diameter of glass beads was around $480 \mu \mathrm{m}$, the mean diameter of particles was $7 \mu \mathrm{m}$, the initial permeability of the medium
Fig. 5 Irregularity of throat radius

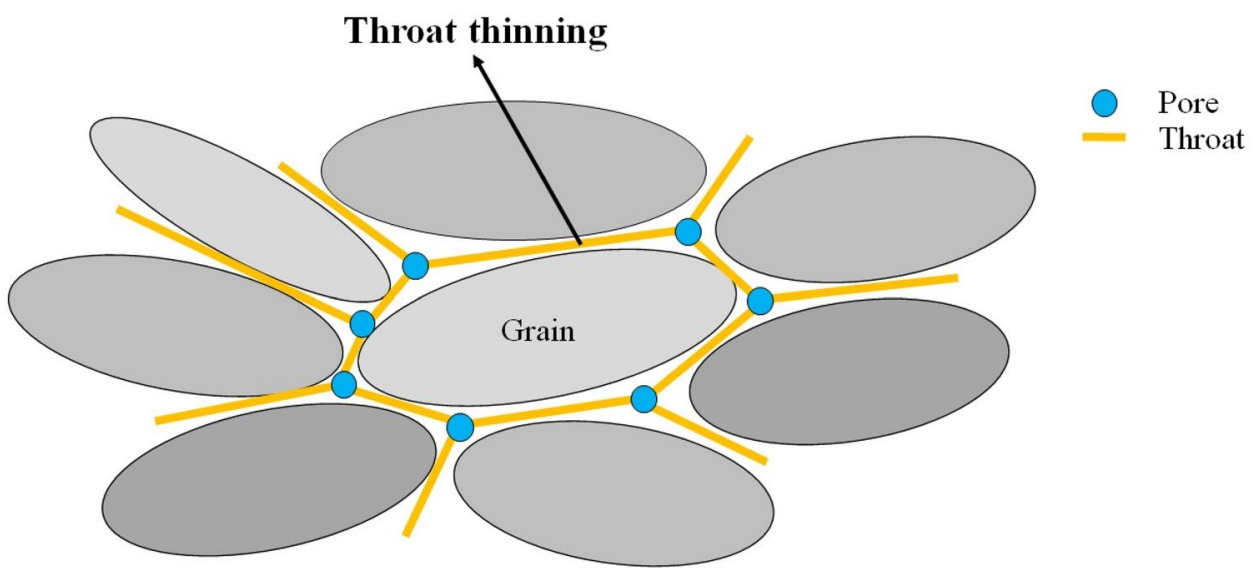

SN Applied Sciences 
was 159 Darcy and the fluid injection rate was $25 \mathrm{cc} / \mathrm{min}$. In order to avoid a huge amount of calculations, only a small piece of the real core is simulated by the pore network. A square shape was selected for the cross-sectional area of the pore network, which is smaller than the core. The special position of the modeled pore network is shown in Fig. 6.

500 pores are selected for this space $\left(1 \times 1 \times 4 \mathrm{~mm}^{2}\right)$. The particles are injected from the interface with two different particle concentrations of $1000 \mathrm{ppm}$ and $2000 \mathrm{ppm}$. The particles are moving through the throats until they get stuck by the straining or the bridging mechanisms, or deposit on the throats surface using surface deposition mechanism. Figure 7 shows a selected section of the 3D pore network after $100 \mathrm{~min}$ of injection. This figure helps to demonstrate the structure of the network and distribution of the channels, pores, throats, and particles. In this figure, the green circles present the pores, the red circles present the moving particles, and the arrows present the throats. The direction of each arrow shows the direction of fluid flow in the throat. It is worth mentioning that Fig. 7 is not representative of the entire 500 pores, as well as, stuck particles. It can be seen in Fig. 7 that the concentration of moving particles in the right hand (fluid inlet) is higher than the left hand of Fig. 7. This is due to the fact that most of the particles are deposited near the inlet face area and a few particles can reach to the outlet.

Moghadasi et al. [4] experimental results are simulated using the proposed model in this study. Subsequently, the permeability of the first $4 \mathrm{~mm}$ of the glass bead is selected for comparison with the obtained results of pore network simulation. Figures 8 and 9 illustrate the dimensionless permeability variation $\left(K / K_{0}\right)$ versus time for injections of particles with the concentrations $1000 \mathrm{ppm}$ and $2000 \mathrm{ppm}$. As shown in these two figures, the results of pore network modeling highly agree with the experimental data. A higher permeability reduction is observed for the case with $2000 \mathrm{ppm}$ particle concentration compared to the case with $1000 \mathrm{ppm}$ particle concentration. This is due to the fact that the presence of more particles in the injection fluid increases the chance of pore-blocking by the mentioned mechanisms.

As can be seen in Figs. 8 and 9, for the total simulation time ( $1600 \mathrm{~min}$ ), $40 \%$ (for the case with $1000 \mathrm{ppm}$ particle concentration) and $60 \%$ (for the case with 2000 ppm particle concentration) of permeability reduction are observed during the first half the simulation time ( $~ 800 \mathrm{~min})$, while permeability reduction is less than $5 \%$ for the rest of the simulation time $(800-1600 \mathrm{~min})$ [4]. It shows in the early times of injection, the particles are blocking the small radius throats that are closer to the interface of porous media, so the permeability of the system is reducing so rapidly. It can be inferred that the area near the injection spot experience a significant blockage and consequently permeability reduction. It can cause severe formation damage and a reduction in the efficiency of the injector. At the end of the injection process, there are only a few throats with a small radius that exist in the outlet of the pore network. This shows that the permeability of the system is changing smoothly at this moment $[4,23,24]$.

As shown here, while the results of modeling show a good agreement with the observation data, the plot of the pore network is not continuous as the observation data. This could be due to the occurrence of unstable flow in the early stage of the modeling, where the particles shifted out of the throat and into the new throat [25]. In the second half of the simulation time (800-1600 $\mathrm{min}$ ), the changes reduced with a more stable reduction of the permeability reduction. On the
Fig. 6 The special position of the modeled pore network

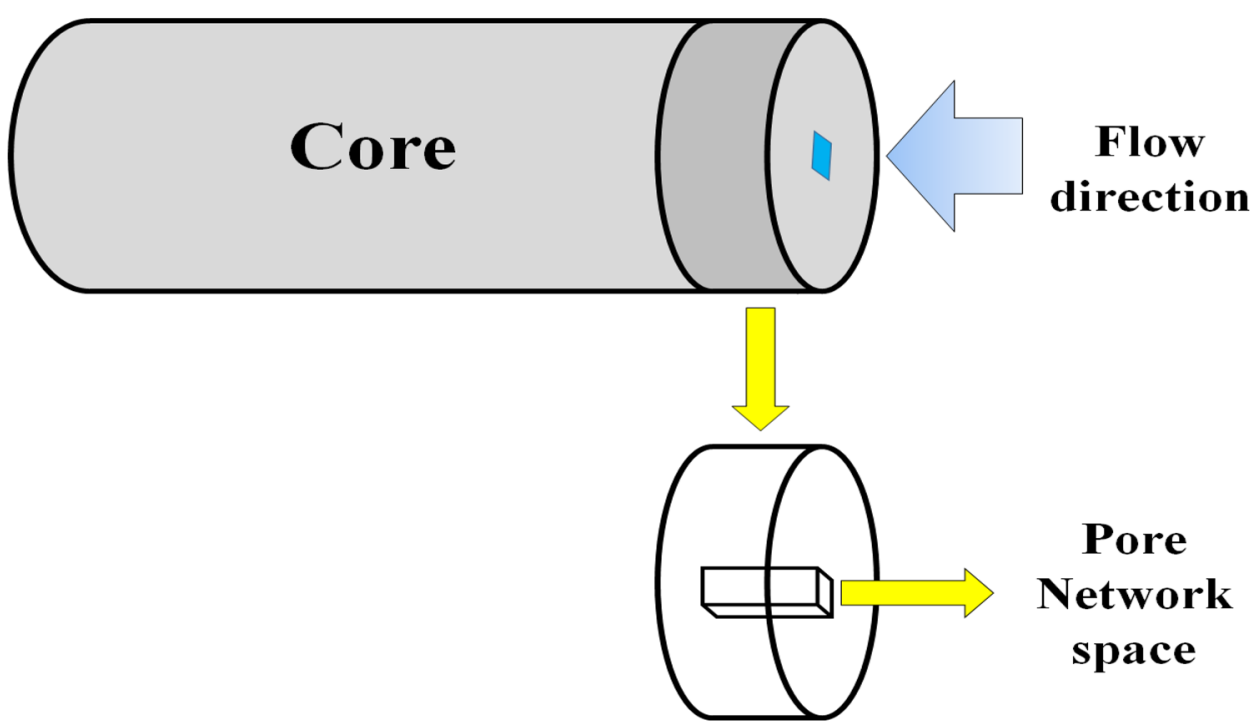




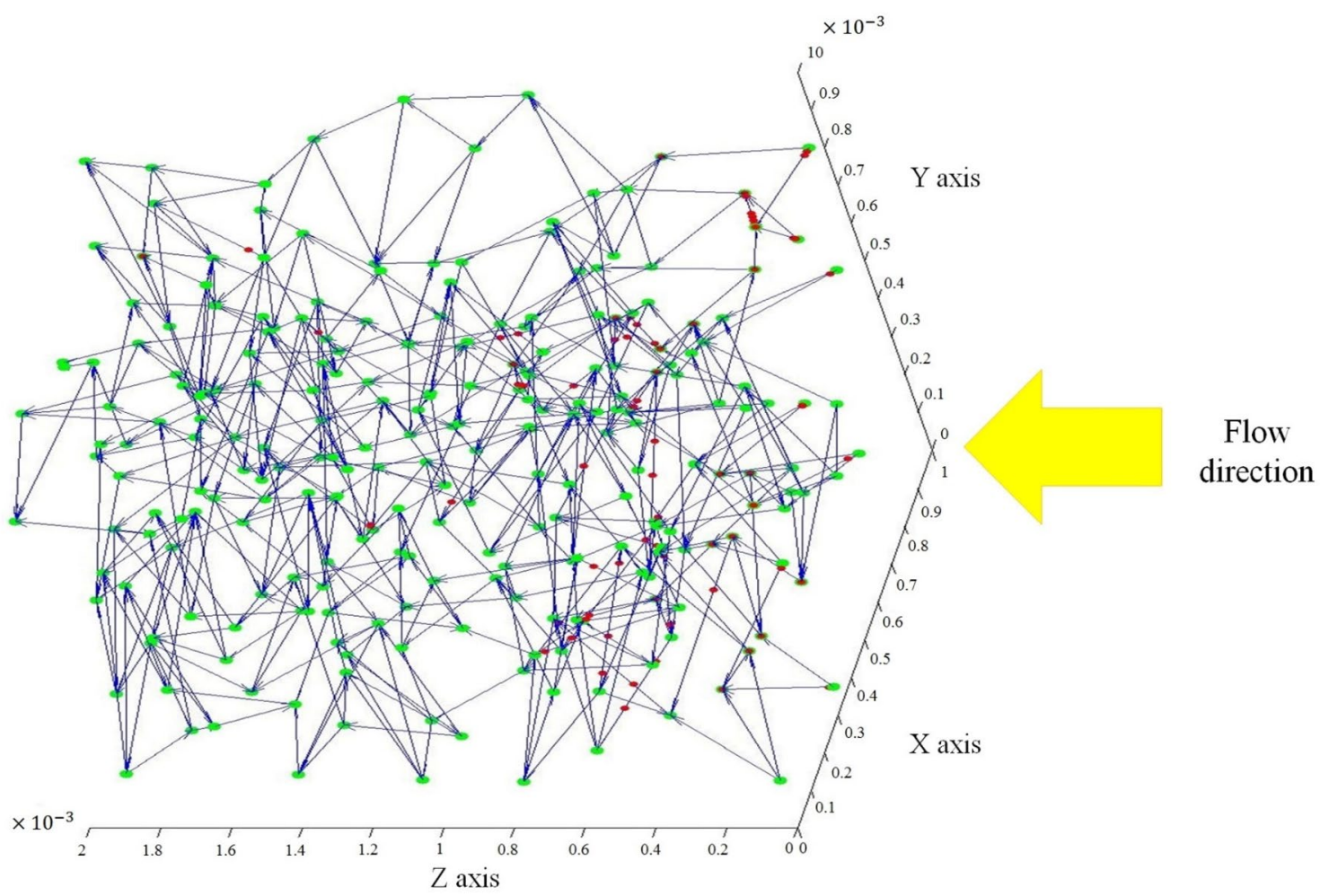

Fig. 7 Particles movement in the presented pore network model after 100 min of injection (The green circles are the pores, the red circles are the particles, and the throats are shown by arrows)

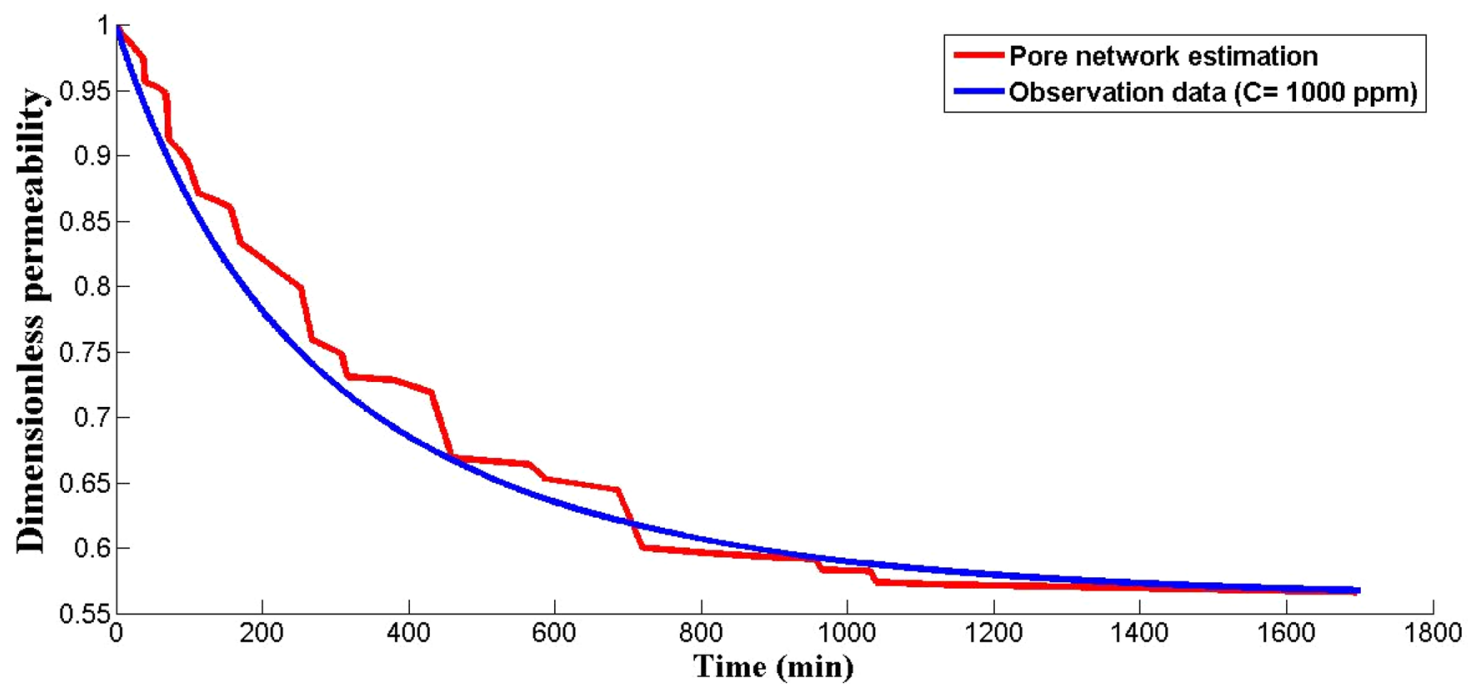

Fig. 8 Permeability reduction in the modeled pore network and observation data ( $C=1000 \mathrm{ppm})$

other hand, when there is a limited number of throats (for example 2000 throats), the permeability of porous media is decreased discretely during a long period of injection (for example $1000 \mathrm{~min}$ ). Only a small amount of throats (about 100-200) were plugged (by the straining or bridging mechanisms) and subsequently, the small value of permeability reduction is not able to make a continuous permeability reduction in the porous media. Furthermore, although the surface deposition is the most highly-occurred particle capturing mechanism, it 


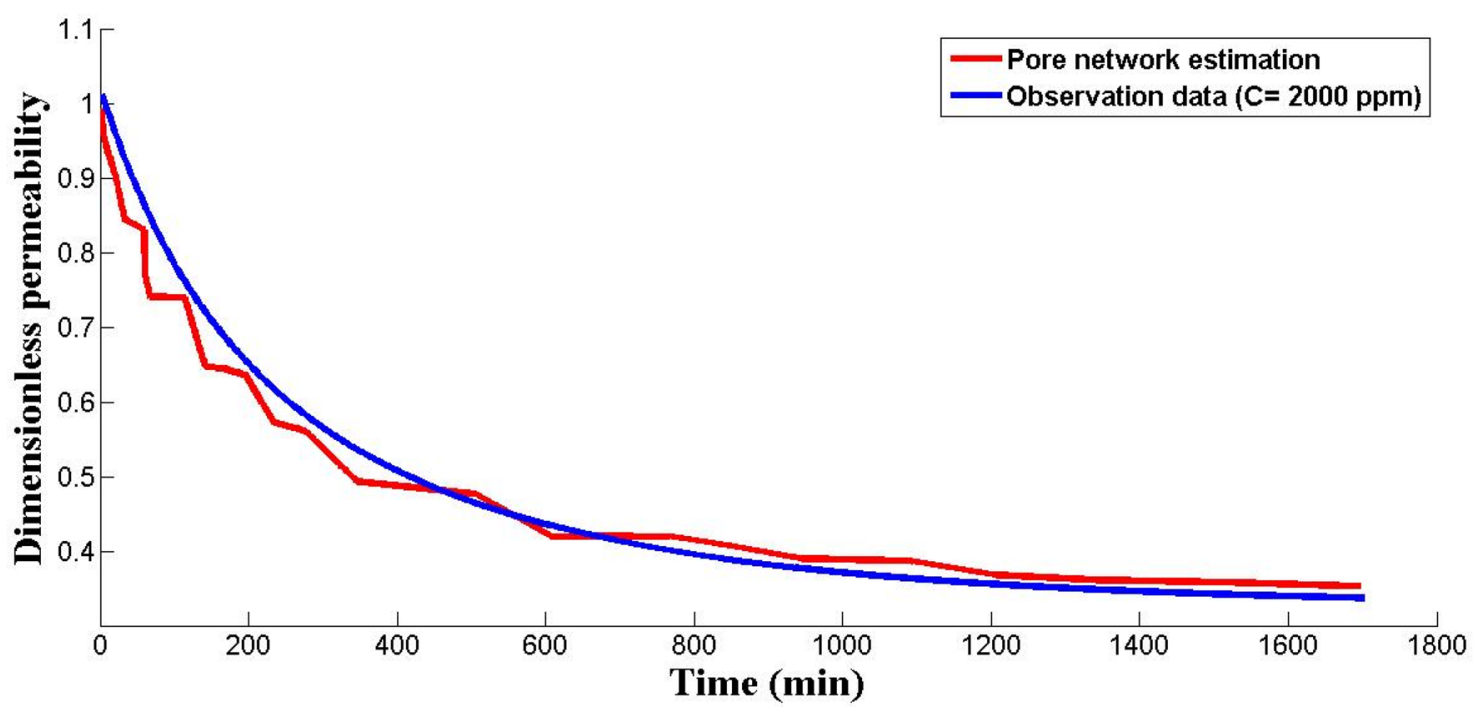

Fig. 9 Permeability reduction in the modeled pore network and observation data ( $C=2000 \mathrm{ppm})$

does not lead to continuous permeability reduction. This is because the permeability reduction caused by surface deposition is not so significant.

\section{Conclusion}

In this study, a 3D pore network model was proposed to simulate the permeability reduction due to the injection of particles to highlight the application of the pore network modeling when deep bed filtration is involved. Surface deposition, straining, and bridging mechanisms were controlling the movement of particles. The severe permeability reduction is observed during the injection of particles, where a higher concentration of the particles causes a higher value of permeability reduction. The obtained results from the pore network are in good agreement with the presented experimental results, which believes this is due to the contribution of the bridging mechanism in the DBF process. This study showed that although the permeability variation of the media is not continuous due to the limited number of pores, a small selected portion of porous media could be the representative of real porous media.

The success of simulation in terms of computational time and cost is highly dependent on the number of pores and selected volume for simulation, which can be named as limitations of this study. In the future, this study can direct to the way, which considers a higher number of pores and stability of the arch.

\section{Compliance with ethical standards}

Conflict of interest The authors declare that they have no conflict of interest.

\section{References}

1. Yang H, Balhoff MT (2017) Pore-network modeling of particle retention in porous media. AIChE J 63(7):3118-3131

2. Oliveira MA et al (2014) Slow migration of mobilised fines during flow in reservoir rocks: laboratory study. J Petrol Sci Eng 122:534-541

3. Bennacer L et al (2013) Suspended particles transport and deposition in saturated granular porous medium: particle size effects. Transp Porous Med 100(3):377-392

4. Moghadasi J et al (2004) Theoretical and experimental study of particle movement and deposition in porous media during water injection. J Petrol Sci Eng 43(3):163-181

5. Todd AC, Somerville J, Scott G (1984) The application of depth of formation damage measurements in predicting water injectivity decline. In: SPE formation damage control symposium. Society of Petroleum Engineers

6. Baghdikian SY, Sharma MM, Handy LL (1987) Flow of clay suspensions through porous media. In: SPE international symposium on oilfield chemistry. Society of Petroleum Engineers

7. Alem A et al (2013) Filtration of kaolinite particles in a saturated porous medium: hydrodynamic effects. Hydrogeol J 21(3):573-586

8. Vaz A et al (2017) Determining model parameters for non-linear deep-bed filtration using laboratory pressure measurements. J Petrol Sci Eng 151:421-433

9. Al-Abduwani FA et al (2005) Formation damage vs. solid particles deposition profile during laboratory simulated PWRI. SPE J 10(02):138-151

10. Herzig J, Leclerc D, Goff PL (1970) Flow of suspensions through porous media-application to deep filtration. Ind Eng Chem 62(5):8-35 
11. Wong R, Mettananda D (2010) Permeability reduction in Qishn sandstone specimens due to particle suspension injection. Transp Porous Med 81(1):105

12. Santos A, Bedrikovetsky P (2006) A stochastic model for particulate suspension flow in porous media. Transp Porous Med 62(1):23-53

13. You Z et al (2014) Asymptotic model for deep bed filtration. Chem Eng J 258:374-385

14. Fatt I (1956) The network model of porous media

15. Rege S, Fogler HS (1988) A network model for deep bed filtration of solid particles and emulsion drops. AIChE J 34(11):1761-1772

16. Burganos V, Paraskeva C, Payatakes A (1992) Three-dimensional trajectory analysis and network simulation of deep bed filtration. J Colloid Interface Sci 148(1):167-181

17. Payatakes AC, Tien C, Turian RM (1973) Part II. Numerical solution of steady state incompressible newtonian flow through periodically constricted tubes. AIChE J 19(1):67-76

18. Ding B, Li C, Wang Y, Xu J (2018) Effects of pore size distribution and coordination number on filtration coefficients for strainingdominant deep bed filtration from percolation theory with 3D networks. Chem Eng Sci 16(175):1

19. French LB (2015) Multiscale modeling of particle transport in petroleum reservoirs
20. Fuchs $R$ et al (2013) Sliding and rolling of individual micrometre sized glass particles on rough silicon surfaces. Tribol Mater Surf Interfaces 7(2):103-107

21. Shi $X$ et al (2013) Coupled solid and fluid mechanics modeling of formation damage near wellbore. J Petrol Sci Eng 112:88-96

22. Civan F (2000) Reservoir formation damage. Gulf Publishing Company, Houston

23. Galaguz YP, Kuzmina LI, Osipov YV (2019) Problem of deep bed filtration in a porous medium with the initial deposit. Fluid Dyn 54(1):85-97

24. Tolmacheva KI, Boronin SA, Osiptsov AA (2019) Formation damage and cleanup in the vicinity of flooding wells: multi-fluid suspension flow model and calibration on lab data. J Petrol Sci Eng 1(178):408-418

25. Zhu W, Li J, Lou Y, Song H (2018) Experiment and capillary bundle network model of micro polymer particles propagation in porous media. Transp Porous Media 122(1):43-55

Publisher's Note Springer Nature remains neutral with regard to jurisdictional claims in published maps and institutional affiliations. 